\title{
BMJ Open Codesigning discharge communication interventions with healthcare providers, youth and parents for emergency practice settings: EDUCATE study protocol
}

To cite: Curran JA, Cassidy C, Bishop A, et al. Codesigning discharge communication interventions with healthcare providers, youth and parents for emergency practice settings: EDUCATE study protocol. BMJ Open 2020;10:e038314. doi:10.1136/ bmjopen-2020-038314

- Prepublication history for this paper is available online. To view these files, please visit the journal online (http://dx.doi org/10.1136/bmjopen-2020038314).

Received 09 March 2020 Revised 14 April 2020 Accepted 17 April 2020

Check for updates

(C) Author(s) (or their employer(s)) 2020. Re-use permitted under CC BY-NC. No commercial re-use. See rights and permissions. Published by BMJ.

For numbered affiliations see end of article.

Correspondence to

Dr Janet A Curran;

jacurran@dal.ca

\section{ABSTRACT}

Introduction Discharge communication is an important aspect of patient care but frequently has shortcomings in emergency departments (EDs). In a paediatric context, youth or parents with young children often leave the ED with minimal opportunity to ask questions or to ensure comprehension of important information. Strategies for improving discharge communication have primarily targeted patients and/or parents, although neither group has been engaged in intervention design or implementation. Furthermore, ED healthcare providers (HCPs), important actors in discharge communication practice, are rarely consulted regarding intervention design decisions. We will generate evidence to enhance discharge communication by engaging youth, parents and HCPs in the codesign of ED discharge communication strategies (EDUCATE) for asthma and minor head injury.

Methods and analysis This mixed methods study will take place at two academic paediatric EDs in Canada. The study will occur in two phases: $(A)$ codesign and refinement of the intervention prototypes; and (B) usability testing of the prototypes. During the first phase, two codesign teams (one for each condition) will follow a series of structured design meetings based on the Behavior Change Wheel to develop the EDUCATE interventions. Each codesign team (composed of youth, parents, HCPs and study researchers) will collaborate to identify priority target behaviours and acceptable components to include in the interventions. During the second phase, we will conduct usability testing in two EDs with a group of youth, parents and HCPs to refine the interventions. Two cycles of usability testing will be conducted with intervention refinement occurring at the end of each cycle.

Ethics and dissemination Informed consent will be obtained from all participants. Ethics approval for this study has been obtained from the Research Ethics Board, IWK Health Centre. Results from this study will form the basis of a future effectiveness implementation trial. Key findings will be presented at national and international
Strengths and limitations of this study

We will use a theory-based systematic method to codesign practice change interventions with youth, parents and healthcare providers.

- This is the first paper to describe a process for codesigning discharge communication interventions with youth, parents and healthcare providers.

- Our protocol describes a process for evaluating the impact of the codesign process as a means for promoting the development of important relationships between healthcare providers, patients and researchers to bridge gaps between research and clinical practice.

- A potential limitation of codesigning interventions with input from key stakeholders is the extra time that may be required to complete the codesign process. This could result in a challenge with retention of participants on the codesign teams. To mitigate these potential challenges, patient engagement experts will be actively involved to support participants throughout our study. Furthermore, to minimise attrition, we aim to provide a tailored orientation programme to prepare all participants for effective collaboration, establish options for asynchronous participation if required and complete the codesign process within 12 months.

conferences and published within peer-reviewed journals.

\section{INTRODUCTION}

More than 4 million children and youth in Canada visit an emergency department (ED) each year, and the vast majority $(87 \%)$ are discharged home under the care of their 
parents. ${ }^{1}$ Discharge communication is a fundamental aspect of high-quality patient-centred care; ${ }^{23}$ however, shortcomings in discharge communication exists across a range of settings and illness presentations. ${ }^{4-8}$ From a narrative review of discharge communication practices, we identified a lack of policies and guidelines supporting discharge communication in Canadian paediatric EDs. ${ }^{9}$ Discharge instructions are often missing important information in both written materials ${ }^{10}$ and verbal instructions. ${ }^{11}$ Furthermore, healthcare providers (HCPs) rarely check for understanding of information that is shared during an ED visit. ${ }^{12}$ This may explain why families leave the ED without fully understanding discharge information. ${ }^{13-16}$ Poor quality discharge communication impacts patient safety and health outcomes, leads to parental stress and can result in the cost of a return visit to the ED. ${ }^{17} 18$

Interventions to improve discharge communication in paediatric EDs vary widely in design and effectiveness. ${ }^{29}$ Few interventions have a theoretical basis and most do not consider the psychological, educational, social or environmental context of the ED. ${ }^{19}$ Poor intervention design is particularly problematic given the communication challenges that can arise from the high stress, unpredictable and dynamic nature of the ED setting. ${ }^{9}$ Parents often leave the ED with minimal opportunity to ask follow-up questions and little information about what to do if questions arise at home. ${ }^{11}$ Previous studies examining parentHCP communication during the ED visit for asthma care showed communication was overwhelmingly biomedical. ${ }^{20}$ Overall, little consideration has been given to the context in which discharge communication is delivered in the ED or how parents use the information at home. ${ }^{21}$ Failure to consider these contextual elements can impact the effectiveness and sustainability of discharge communication interventions.

Despite being key actors in the discharge communication process in a paediatric emergency care context, youth, parents/caregivers and HCPs have not been involved in the intervention design process. ${ }^{21}$ Codesigning healthcare services has the potential to challenge existing hierarchical structures in healthcare that often favour system-centred design over patient-centred design.

Partnering with youth, parents and HCPs is an essential aspect of health redesign ${ }^{62}$ and presents a unique opportunity to improve communication, quality of care, cost efficiency and population health. ${ }^{23}$ Interventions that engage youth and parents can improve adherence to recommended treatments and lead to more sustainable and appropriate interventions. ${ }^{24-26}$ Codesign initiatives can also help foster the development of important relationships between HCPs and researchers to bridge gaps between research and clinical practice, promoting partnerships early in the research process to optimise knowledge uptake in practice. ${ }^{27}$

We have chosen two different ED presentations, asthma and minor head injury, to evaluate a codesign method for discharge communication interventions. Asthma and minor head injury provide a useful context to test feasibility of our codesign strategy, as they are common presentations in paediatric EDs. Patients and families are more likely to have previous presentations in the ED for asthma than minor head injury. However, it is not clear how previous knowledge or experience with an illness presentation in the ED can influence patient, parent or HCP discharge communication behaviours. Furthermore, the assessment and management of both presentations are well supported by national guidelines. ${ }^{28-33}$ Thus, these two illness presentations provide rich opportunities to explore different stakeholder perspectives.

Given the wide variation and limitations in discharge communication practices, the lack of inclusion of key stakeholders in designing discharge communication interventions and the consequences of poor discharge communication, this study is of high importance. Our study will generate evidence to improve discharge communication in paediatric emergency care by collaborating with youth, parents and HCPs to codesign acceptable and effective discharge communication strategies.

\section{METHODS AND ANALYSIS \\ Aim and objectives}

The primary aim of our study is to generate evidence to improve discharge communication in paediatric emergency care. We will achieve this aim through the following objectives:

1. Codesign two Emergency department Discharge commUniCation strATEgies (EDUCATE); one for asthma (A-EDUCATE) and another for minor head injury (MHI-EDUCATE).

2. Test the usability of the codesigned interventions.

\section{Setting}

This study is endorsed by Pediatric Emergency Research Canada (PERC) and will take place in two paediatrics EDs located in urban academic paediatric tertiary care centres in two Canadian provinces.

\section{Design}

We will employ a mixed method study design ${ }^{34}$ that draws on the Behavior Change Wheel (BCW) ${ }^{35}$ to systematically understand barriers, behaviours and context features relevant to discharge communication. ${ }^{36}$ The BCW is based on a comprehensive analysis of behaviour; it explores which internal and external conditions need to be in place for an individual to achieve a specific behavioural target (eg, make follow-up appointment with family physician). ${ }^{37}$ Central to the BCW is the Capability, Opportunity, Motivation and Behavior model, which proposes that understanding 'behaviour' in context requires examining the 'capability' to perform the behaviour, the 'opportunity' for the behaviour to occur and the strength of one's 'motivation' to enact the behaviour. ${ }^{37}$

This study will be carried out in two phases between December 2019 and May 2021; phase A (codesigning and 
refining) and phase B (usability testing). The codesign process will take place at one paediatric ED and usability testing will occur at two sites. While the study population, study design, outcome measures and analysis are described separately in this protocol, the process is understood to be iterative given the overlap with the refinement procedure and usability testing of the interventions.

\section{Phase A: codesign and refinement of the EDUCATE interventions \\ Study population and recruitment}

Two design teams will be established at the IWK Health Centre: one for the A-EDUCATE and another for the MHI-EDUCATE interventions. Each design team will consist of three youths, three parents and four ED HCPs. Representation of HCPs on each design team will include at least one nurse and one physician currently working in a paediatric ED. Composition of the design teams was purposely chosen to allow for unexpected absentees while maintaining sufficient representation from each knowledge user group, as well as to ensure adequate peer support for youth and parent participants at each meeting. Youth and parents will be eligible to participate if they speak English and have previous experience in an ED. Youth were purposely included as an important stakeholder group since adolescence is a time of exploring autonomy and youth can present to the ED without parents or caregivers. ${ }^{38}$ Youth will be considered any individual $12-17$ years of age.$^{39}{ }^{40}$ Factors such as English as a second language and low literacy scores are known to influence comprehension, implementation and adherence to discharge instructions. ${ }^{41}$ Therefore, we will use maximum variation sampling ${ }^{42}$ along the following parameters to recruit study participants: health literacy level, geographic location, English as a second language and gender. Health literacy level will be determined during screening using the METER. ${ }^{43}$ All screening for study eligibility will be completed using an online questionnaire via REDCap, ${ }^{44}$ an electronic data capture tool. Recruitment of youth and parent participants will happen through social media advertisements and posters in the ED. Recruitment of HCP participants will occur through institutional email invitation. Study recruitment will continue until the required representation for each design team is reached. Each design team will also be supported by a patient engagement specialist, a knowledge translation researcher with expertise in intervention design, a content expert (asthma or minor head injury) and a human performance technologist. All codesign meetings will be cofacilitated by a parent and a researcher.

\section{Codesign procedure}

Both design teams will follow a meeting schedule based on the BCW guide that reflects a systematic procedure for intervention design (figure 1). Using this theoretical approach, teams will be guided in a systematic and transparent way to identify intervention components that are connected to important behavioural targets. Teams will also be provided with information on typical ED patient flow and care processes to assist with consideration of the impact of discharge communication interventions on factors such as staff workload, length of stay or resources. ${ }^{45} 46$

Each team will meet a total of six times over 12 months, and meetings will last approximately 2 hours. Youth with chronic illness and their families have demonstrated a willingness to participate in intervention design following a similar time commitment of eight 2-hour workshops over 11 months. ${ }^{47}$

A number of communication strategies will be used to keep the design teams and the research team informed throughout the study. Regular email progress updates will be used to communicate with design team members between meetings. Youth, parents and HCPs will also have access to online or telephone support between meetings to address any questions or concerns that may arise during the design process. A research assistant will attend all design team meetings to observe the group process and track all decisions made at each meeting. Design team meeting notes will be reviewed regularly with the research team to identify challenges and track consistency between the two groups. Lastly, to encourage attendance and participation, the option for teleconferencing will be

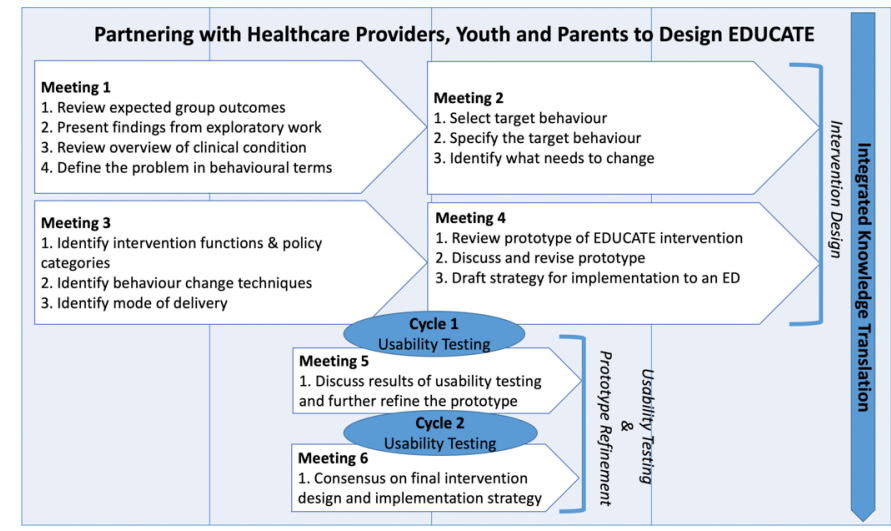

Figure 1 Intervention design method. ED, emergency department; EDUCATE, emergency department discharge communication strategies. 
made available to anyone unable to attend design team meetings in person.

Prior to attending the initial meeting, design team members will complete a tailored orientation programme to prepare them for effective collaboration through the codesign process. Content and delivery of the orientation training will be based on the findings of a needs assessment completed by design team members. The training will be customised to meet each groups' specified learning needs, preferred learning modalities and readiness to participate in the codesign process.

\section{Intervention design (meetings 1-4)}

Development of the draft prototype of the EDUCATE interventions will occur over the first four meetings. The teams will use a nominal group technique to achieve consensus on all design decisions. ${ }^{48}$ In each meeting, the team will review a meeting agenda and reaffirm the purpose of the codesign approach. At the first meeting, cochairs (parent and HCP) will be identified for each codesign team, and all team members will be asked to sign a confidentiality agreement. The goal of the first meeting is to begin to understand the particulars of the problem. The facilitator will start the meeting by sharing the key findings from exploratory work identifying the barriers and enablers for discharge communication. ${ }^{21}$ A content expert will also attend the first meeting and provide an overview of the clinical condition (asthma or minor head injury). Each design team will use the collective information and their experience to describe the problem in behavioural terms and specify the range of targets (eg, interruptions in communication and adherence to controller medications) relevant to discharge communication for the clinical condition. Each of the target behaviours will then be specified according to who needs to do what differently, when, where, how and with whom. ${ }^{49}$

At meeting 2, the design teams will take part in a priority setting exercise using the full list of target behaviours generated in meeting 1 . Considering the barriers and enablers for discharge communication reviewed during the first meeting, the participants will assess each of the target behaviours using the following questions: (1) what is the likely impact if the target behaviour is changed; (2) how easy will it be to change the target behaviour; (3) how relevant is the target behaviour to improving discharge communication; and (4) is the behaviour change measurable? The teams will then use the Affordability, Practicability, Effectiveness/cost-effectiveness, Acceptability, Side-effects/safety and Equality (APEASE) criteria $^{35}$ to identify priority target behaviours.

During meeting 3 , the design teams will begin to storyboard $^{50}$ or create a series of images used to depict a narrative for the purpose of previsualising the prototypes. Storyboarding the EDUCATE interventions will occur by working through a set of structured tasks. The first task will be to link the priority target behaviours with the corresponding intervention function (ie, education, training, persuasion and environmental restructuring) and policy category (ie, guidelines, legislation and service provision) from the BCW. ${ }^{35}$ The design teams will consider the target behaviours and the context in which the behaviour occurs when making their choices. Next, the design teams will select the specific components of the intervention (eg, feedback on behaviour and remove adverse stimulus) through a mapping exercise that includes consideration of relevant barriers and a list of 93 possible behaviour change techniques (BCTs) from the BCT Taxonomy v1. ${ }^{51}$ The final step will include selecting the mode of delivery (eg, face to face, written and video) for each of the components. A human performance technologist will also attend meeting 3 and guide each design team through a series of instructional design tasks to vet possible intervention modalities and delivery solutions (eg, paper based, use of media and web-based). After meeting 3, the research team and the instructional designer will use the meeting notes from each of the design teams to develop a prototype for the A-EDUCATE and MHI-EDUCATE interventions.

Each design team will reconvene for meeting 4 to review the prototype of the A-EDUCATE and MHI-EDUCATE interventions and identify any necessary revisions. The design teams will again evaluate the prototype using the APEASE criteria. ${ }^{35}$ As a final task, each design team will develop a draft implementation plan for their EDUCATE interventions.

\section{Prototype refinement (meetings 5 and 6 )}

Prototype refinement will occur during design team meetings 5 and 6 . These meetings will occur during the usability testing phase. In meeting 5 , feedback collected from cycle 1 of usability testing at both study sites will be summarised and presented to each design team. Considering the new information from user testing, the design teams will be instructed to refine the prototypes adhering to the APEASE criteria. The refined prototypes will be evaluated in cycle 2 of usability testing. Results gathered from cycle 2 of the usability testing at both sites will be implemented to finalise the EDUCATE interventions. Meeting 6 will conclude with consensus on the final product of the EDUCATE interventions and the implementation strategy.

\section{Outcome measures}

We will capture process and summative data to describe and evaluate the experience of the codesign teams. At the end of each design team meeting, participants will be asked to complete a brief survey using Likert-type scales that rate the characteristics of successful patient, parent and HCP engagement including: inclusive mechanisms and processes, multiway capacity building, multiway communication, experiential knowledge valued as evidence and shared sense of purpose ${ }^{52}$ Additional questions will be adapted from the validated Patient Engagement \& Evaluation Tool ${ }^{53}$ and used to assess participants' self-perceived engagement during each design meeting. 
Design team participants will also be invited to document their experiences over the 12 months in a journal log. Similarly, a research assistant will maintain a $\log$ throughout the design process to capture observations related to the intervention design meetings (eg, attendance, length of meetings, decisions regarding important barriers and enablers, selection of BCTs and mode of deliver for the intervention, presence of any decisional conflicts and quality/quantity of participation by all individuals on the design teams). This process data will be important to inform our understanding of how and why the codesign process worked or did not work.

Summative data will be collected at the end of the codesign process. After meeting 6, a member of the research team not involved in the codesign procedure will conduct brief individual interviews with members of the design teams to gather additional information about their overall experience. Our approach for soliciting youth, parent and HCP input will be based on the Guide for Capturing Patient, Public and Service User Feedback Effectively. ${ }^{54}$ We will use open-ended questions to probe participants' perspectives about the BCW as a method to guide intervention design; beliefs and attitudes regarding the inclusion of youth, parents and HCPs in the design process; perceived barriers to participating in the experience; and suggestions for the design of future discharge communication interventions. All interviews will be audio-recorded, transcribed verbatim and deidentified prior to analysis. To complement the individual interviews, we will capture detailed data on the quality of patient engagement using the Patient Engagement in Research Scale. ${ }^{55}$

\section{Analysis}

Process and interview data will be managed using NVivo qualitative data analysis software (QSR International Pty Ltd, Version 10, 2014). We will use a conventional content analysis approach ${ }^{56}$ to identify themes and subthemes relevant to youth, parent and HCP roles and contributions in the intervention design process. We will report ratings for successful engagement and specific types of challenges (eg, decision conflict and task completion) using either parametric or non-parametric descriptive statistics with CIs, depending on the nature of the distribution of the data once collected.

\section{Phase B: usability testing of EDUCATE interventions}

\section{Study design}

The prototypes of the EDUCATE interventions developed during design team meetings 1-4 will undergo usability testing in phase B. Testing the usability of interventions prior to implementation can help to identify problems with acceptability, compliance and inform the implementation strategy. ${ }^{57}$ Each intervention will undergo two cycles of usability testing at both study sites with participants who were not involved with the intervention design process.

Usability testing will involve observations and interviews in iterative cycles to assess the usability of the EDUCATE interventions and further refine the prototypes. ${ }^{58} 59$ Scenarios involving structured tasks will be developed based on the characteristics of each prototype to fully test their functionality. During the observation sessions, participants will be provided with a private space to work through the structured tasks aligned with the EDUCATE interventions. To cover the major functionalities of the intervention, we will use a cognitive task analysis approach ${ }^{57}$ where users are asked to think aloud as they perform the specific tasks outlined in the scenario. ${ }^{60}$ The think aloud exercises, which will take $30-45 \mathrm{~min}$, will be audio recorded. All user data will be anonymised, tracked, logged and summarised to share with the design teams. Feedback from cycle one of the usability testing will be presented to the design teams in meeting 5 for prototype refinement. The refined prototypes will be evaluated in cycle 2 of usability testing at both ED sites. Results from cycle 2 of usability testing will be given to the design teams at meeting 6 and will be used to finalise the EDUCATE interventions.

\section{Study population and recruitment}

Recruitment for the usability testing will occur through social media, letters of invitation and posters in the ED waiting rooms at both study sites. Each testing session will involve youth, parents and HCPs. To ensure a diverse sample of youth and parents for each cycle of usability testing, we will use the same maximum variation recruitment strategy as described above in the first phase of the study. Eligible youth and parent participants will have presented to the paediatric ED at either of the study hospitals in the last month with asthma or a minor head injury. HCP participants will include nurses and physicians who care for children in the ED. Exclusion criteria will include all members of the design teams and nonEnglish speaking individuals. Previous usability studies have found that three to four participants is sufficient to find $80 \%$ of design usability problems and exhaustive tests with larger samples are counterproductive, particularly in the early design phase ${ }^{61}$ Therefore, two youth, two parents and four HCPs will be recruited at each hospital site per intervention and per cycle of testing.

\section{Outcome measures}

Data will be captured on the following metrics: usability problems, total time required to complete tasks, errors and any concerns observed or expressed during the think aloud exercise. ${ }^{61}$ We will also gather user satisfaction at the end of the usability test using a validated quantitative assessment survey to evaluate five domains (purpose, appearance, usefulness, overall impact and content) for parents (15 items) and HCPs (30 items). ${ }^{62}$ Youth will complete the parent user survey.

\section{Analysis}

Transcripts from the think aloud recordings will be managed in NVivo qualitative software. Content analysis will be used to code qualitative data according to each of 
the cognitive tasks outlined in the usability test. Thematic analysis will be used to group user problems and concerns verbalised during task completion. All quantitative usability data will be analysed in SPSS software (V.22). Relevant descriptive statistics will be generated to describe demographic characteristics, usability problems and user satisfaction. Data from both phases will be triangulated $^{63}$ to assist with the development of an explanatory framework for understanding the codesign experience. A matrix structure will be developed for better visualisation of converging and conflicting data.

\section{Patient and public involvement}

Two parents served as patient and family representatives throughout the conceptualisation of this study. Both parents were identified on the submission for research funding, as a principal knowledge user and a coapplicant, respectively. The IWK Family Leadership Council, the IWK Youth Advisory Council and the patient engagement coordinator from the Maritime Strategy for Patient Oriented Research SUPPORT Unit (MSSU) have also contributed to the planning of this project. Our parent partners will be involved in the interpretation and dissemination of our study results.

\section{ETHICS AND DISSEMINATION}

This study protocol has been reviewed and approved by the institutional research ethics board at the IWK (REB \#1024004) on 18 December 2018. All youth, parent and HCP participants will be asked to provide consent prior to their involvement in the study. Additional REB approval will also be obtained from the second study site.

Our dissemination plan will include a multipronged approach. First, we will leverage our existing collaborations and networks to disseminate key findings to stakeholder groups (patient advocacy groups, parents, ED researchers, research administrators and funders). Our current linkages and partnerships with groups such as the MSSU, PERC and the Translating Emergency Knowledge for Kids network provide us with national and international dissemination opportunities. PERC has linkages with international paediatric emergency care research nodes in the USA, Europe and Australia through the global Pediatric Emergency Research Networks association. We will disseminate key findings to node leaders through existing portals. We will also present our findings at national and international meetings and publish in open access journals.

\section{DISCUSSION}

Codesign is recognised as an important and effective approach for strengthening interventions that target healthcare practices. ${ }^{64-73}$ Successful discharge communication in ED practice requires the active involvement of HCPs, parents or caregivers and patients. Including these relevant stakeholders in the design and usability testing of interventions to support discharge communication is particularly important given the interventions are likely to involve behaviour change. ${ }^{74}$ Our study is distinct with respect to its engagement of youth, parents and HCPs to account for all participant perspectives in the design of discharge communication interventions. To explore various stakeholder perspectives, we will codesign interventions for asthma and minor head injury, which are common ED presentations with differing assessment and management practices. Furthermore, our study uses a theory-based design procedure, which will allow us to conceptually model the intervention components and make explicit the pathways or mechanisms that will lead to the desired outcomes identified by youth, parents and HCPs. This approach aligns with the Medical Research Council framework for the development and evaluation of complex interventions. ${ }^{75}$

While the diversity of our study population is a strength, we acknowledge there may be challenges with bringing together three very different stakeholders groups with busy schedules, competing personal commitments and varying motivation to participate. In particular, the retention of youth, parent and HCP participants for the duration of the intervention design phase will be important. We have developed a robust strategy to help mitigate potential challenges by involving patient engagement experts at all stages of the project and designing a comprehensive orientation training programme to enhance participants' understanding of the codesign process and to support relationship building. We also anticipate that participants will be intrinsically motivated to see the codesign work through to completion when they realise the significant role they can play in health system change. ${ }^{76}$ Further strategies for participant retention include honoraria and compensation for childcare, parking, travel and other associated costs.

Literature on codesign in healthcare settings has also cautioned against potential conflicts related to power dynamics, ${ }^{22} 7778$ such as emerging struggles between patients, parents and HCPs. Given that many patients or parents have traditionally taken on a more passive or subordinate role in their relationships with HCPs, some participants may require support to voice their opinions in a room with medical professionals. In particular, youth may find the task of participating as equals on the design team difficult, especially given the age differential with other team members. To help participants feel more comfortable, we have included three youth and three parents on each design team. Furthermore, each design team will include a greater number of youth and parents to HCPs. Beyond facilitating rapport building, our orientation training programme will also strive to empower all participants to work together as mutual partners.

To date, youth, parent and HCP stakeholders have not be involved in designing discharge communication interventions or identifying relevant outcomes. ${ }^{21}$ Findings from our work will make a valuable contribution to the intervention design and paediatric emergency care literature. Our 
evidence-based approach to develop the two EDUCATE interventions will be the first Canadian study to design and evaluate paediatric emergency discharge communication interventions in real partnership with youth, parents and HCPs. The refined EDUCATE interventions will be evaluated in a future trial and have the potential to influence the design of future discharge communication practice in paediatric EDs. Through enhancing discharge communication, this study may ultimately impact a critical patient safety risk, improve patient agency in their own healthcare, improve health outcomes and lead to better utilisation of ED resources. Finally, insights gained from our codesign approach involving youth, parents and HCPs will provide guidance for other teams wanting to engage families in research.

\section{Author affiliations}

${ }^{1}$ Department of Nursing, Dalhousie University, Halifax, Nova Scotia, Canada ${ }^{2}$ Department of Pediatrics, IWK Health Centre, Halifax, Nova Scotia, Canada ${ }^{3}$ IWK Health Centre, Halifax, Nova Scotia, Canada

${ }^{4}$ Nova Scotia Health Authority, Halifax, Nova Scotia, Canada

${ }^{5}$ Department of Pediatrics, University of Ottawa, Ottawa, Ontario, Canada

${ }^{6}$ Faculty of Education, Mount Saint Vincent University, Halifax, Nova Scotia, Canada

${ }^{7}$ Li Ka Shing Knowledge Institute, Saint Mike's, Toronto, Ontario, Canada

${ }^{8}$ Department of Pediatrics, University of Alberta, Edmonton, Alberta, Canada

${ }^{9}$ Division of Emergency Medicine, Children's Hospital of Eastern Ontario, Ottawa,

Ontario, Canada

${ }^{10}$ Department of Pediatrics, CHEO, Ottawa, Ontario, Canada

${ }^{11}$ Clinical Epidemiology Program, Ottawa Health Research Institute, Ottawa, Ontario, Canada

Acknowledgements We would like to thank our parent partners for their contributions on this project and to the research team. In addition, we would like to acknowledge that this study is endorsed by Pediatric Emergency Research Canada (PERC), which is a network of healthcare professionals formed to conduct multicentre research projects. Authors JAC, ACP, MJ, SM, EB and RZ are current members of PERC. For more information about PERC, please contact Rebecca. emerton@albertahealthservices.ca.

Contributors JAC is the principal investigator. She conceptualised and designed this study, prepared the grant application and obtained funding. CC, AB and LW made substantial contributions to the development of the grant proposal for funding. CC and LW will assist with facilitating codesign meetings. ACP provided insight for the grant proposal and will be involved with the usability testing at the second study site. KR offered guidance on statistical analysis for this manuscript, and she will take part in the final data analyses and interpretation. SES contributed substantial feedback to the grant proposal and will assist with development of the training/orientation programme for participants on the codesign teams. HW prepared the first draft of this manuscript. She will coordinate the study and assist with data collection. AN, MJ, SM, SB, EB, JC, JL, MD, RM, RZ and TP provided important support for the grant application. MJ, SM, EB and RZ will contribute input for creating the scenarios for usability testing. JL and RM are the parent/patient knowledge users on the grant and will provide guidance during codesign meetings. JG contributed significantly to the conception of the study and will assist with interpretation of the final data. PERC will endorse this study. All authors critically appraised the intellectual content of the manuscript and provided input and revisions. All authors read and approved the final manuscript.

Funding This study was funded by the Canadian Institutes of Health Research, Project Grant (Application \#399798).

Competing interests None declared.

Patient and public involvement Patients and/or the public were involved in the design, or conduct, or reporting, or dissemination plans of this research. Refer to the Methods section for further details.

Patient consent for publication Not required.

Ethics approval Ethics approval for this protocol (version date 10 December 2019) was granted by the institutional research ethics board at the IWK Health
Centre (REB \#1024004). Written informed consent will be obtained from all study participants.

Provenance and peer review Not commissioned; peer reviewed for ethical and funding approval prior to submission.

Open access This is an open access article distributed in accordance with the Creative Commons Attribution Non Commercial (CC BY-NC 4.0) license, which permits others to distribute, remix, adapt, build upon this work non-commercially, and license their derivative works on different terms, provided the original work is properly cited, appropriate credit is given, any changes made indicated, and the use is non-commercial. See: http://creativecommons.org/licenses/by-nc/4.0/.

ORCID iD

Janet A Curran http://orcid.org/0000-0001-9977-0467

\section{REFERENCES}

1 Canadian Institute for Health Information. Who is visiting the emergency department and how long are they waiting, 2007. Available: https://secure.cihi.ca/free_products/Wait_times_e.pdf [Accessed 23 May 2019].

2 Samuels-Kalow ME, Stack AM, Porter SC. Effective discharge communication in the emergency department. Ann Emerg Med 2012;60:152-9.

3 Institute for Patient and Family-Centered Care. Advancing the practice of patient- and family-centered care in hospitals. How to get started, 2015. Available: http://www.ipfcc.org/pdf/getting_started.pdf [Accessed 23 May 2019].

4 Taylor DM, Cameron PA. Discharge instructions for emergency department patients: what should we provide? J Accid Emerg Med 2000;17:86-90.

5 Hawley CA, Unsworth L, Qureshi KL, et al. Discharge advice provided by UK emergency departments after minor head injury in children. Brain Inj 2012;26:309-799.

6 Johnson B, Abraham M, Conway J, et al. Partnering with patients and families to design a patient and family-centered health care system. Bethesda MD: Institute for Family-Centered Care, 2008.

7 Chorley JN. Ankle sprain discharge instructions from the emergency department. Pediatr Emerg Care 2005;21:498-501.

8 Samuels-Kalow M, Rhodes K, Uspal J, et al. Unmet needs at the time of emergency department discharge. Acad Emerg Med 2016;23:279-87.

9 Curran J. Discharge instructions for parents in the context of pediatric emergency care: a narrative review. BMC Health Serv Res 2014;14:P20.

10 Osborne ZP, Bryant SM. Patients discharged with a prescription for acetaminophen-containing narcotic analgesics do not receive appropriate written instructions. Am J Emerg Med 2003;21:48-50.

11 Vashi A, Rhodes KV. "Sign right here and you're good to go": a content analysis of audiotaped emergency department discharge instructions. Ann Emerg Med 2011;57:315-22.

12 Curran J, Bishop A, Plint A. Understanding discharge communication behaviours in pediatric emergency care. Paediatr Child Health 2018;23:e8-9.

13 Waisman Y, Siegal N, Siegal G, et al. Role of diagnosis-specific information sheets in parents' understanding of emergency department discharge instructions. Eur J Emerg Med 2005;12:159-62.

14 Joseph D, Lee R, Tibbo-Valeriote $\mathrm{H}$, et al. Characteristics and Discharge Instructions for School Aged Patients Evaluated in a Community-Based Level I Trauma Center Children's Emergency Department After Mild Traumatic Brain Injury. Acad Emerg Med 2019;18:S163.

15 Stevens PK, Penprase B, Kepros JP, et al. Parental recognition of postconcussive symptoms in children. $J$ Trauma Nurs 2010;17:178-82.

16 Samuels-Kalow ME, Stack AM, Porter SC. Parental language and dosing errors after discharge from the pediatric emergency department. Pediatr Emerg Care 2013;29:982-7.

$17 \mathrm{Ng} \mathrm{CP}$, Chung $\mathrm{CH}$. An analysis of unscheduled return visits to the accident and emergency department of a general public hospital. Hong Kong J of Med 2003;10:153-61.

18 Kuan WS, Mahadevan M. Emergency unscheduled returns: can we do better? Singapore Med J 2009;50:1068-71.

19 Canadian Institute for Health Information. Canadian emergency department Trends- April 1, 2012 to March 31, 2013, 2014. Available: https://www.cihi.ca/en/nacrs_infosheet_feb2014_en.pdf [Accessed 23 May 2019]. 
20 Wissow LS, Roter D, Bauman LJ, et al. Patient-Provider communication during the emergency department care of children with asthma. Med Care 1998;36:1439-50.

21 Curran JA, Gallant AJ, Zemek R, et al. Discharge communication practices in pediatric emergency care: a systematic review and narrative synthesis. Syst Rev 2019;8:83.

22 Donetto S, Pierri P, Tsianakas V, et al. Experience-Based Co-design and healthcare improvement: Realizing participatory design in the public sector. Des J 2015;18:227-48.

23 Bate P, Robert G. Experience-Based design: from redesigning the system around the patient to co-designing services with the patient. Qual Saf Health Care 2006;15:307-10.

24 Shen S, Doyle-Thomas KAR, Beesley L, et al. How and why should we engage parents as co-researchers in health research? A scoping review of current practices. Health Expect Int J Public Particip Health Care Health Policy.

25 Street RL, Makoul G, Arora NK, et al. How does communication heal? pathways linking clinician-patient communication to health outcomes. Patient Educ Couns 2009;74:295-301.

26 Gagliardi AR, Légaré F, Brouwers MC, et al. Patient-mediated knowledge translation (PKT) interventions for clinical encounters: a systematic review. Implement Sci 2016;11:26.

27 Roberge-Dao J, Yardley B, Menon A, et al. A mixed-methods approach to understanding partnership experiences and outcomes of projects from an integrated knowledge translation funding mode in rehabilitation. BMC Health Serv Res 2019;19:230.

28 Becker $A$, Lemière $C$, Bérubé $D$, et al. Summary of recommendations from the Canadian asthma consensus guidelines, 2003. CMAJ 2005;173:S3-11.

29 Lougheed MD, Leniere C, Ducharme FM, et al. Canadian thoracic Society 2012 guideline update: diagnosis and management of asthma in preschoolers, children and adults: Executive summary. Can Respir J 2012;19:e81-8.

30 Osmond MH, Klassen TP, Wells GA, et al. Catch: a clinical decision rule for the use of computed tomography in children with minor head injury. Can Med Assoc J 2010;182:341-8.

31 Pickering A, Harnan S, Fitzgerald P, et al. Clinical decision rules for children with minor head injury: a systematic review. Arch Dis Child 2011;96:414-21.

32 Kuppermann N, Holmes JF, Dayan PS, et al. Identification of children at very low risk of clinically-important brain injuries after head trauma: a prospective cohort study. Lancet 2009;374:1160-70.

33 CDC. Heads up. cent. Dis. control Prev, 2019. Available: https:// www.cdc.gov/headsup/index.html [Accessed 23 May 2019].

34 Creswell J, Clark P V. Designing and conducting mixed methods research. 2nd ed. Los Angeles; London: SAGE, 2011.

35 Michie S, van Stralen MM, West R. The behaviour change wheel: a new method for characterising and designing behaviour change interventions. Implement Sci 2011;6:42.

36 Glanz K, Rimer B, Viswanath K. Health behavior and health education: theory, research, and practice. 4th Edition. San Francisco, California: John Wiley \& Sons, 2008.

37 Michie S, Atkins L, West R. The behaviour change wheel: a guide to designing interventions. 1st edn. Great Britain: Silverback Publishing, 2014.

38 Benjamin L, Ishimine $\mathrm{P}$, Joseph $\mathrm{M}$, et al. Evaluation and treatment of minors. Ann Emerg Med 2018;71:225-32.

39 Government of Canada SC. Measured youth body mass index (BMI), 2017. Available: https://www150.statcan.gc.ca/t1/tbl1/en/tv.action? pid $=1310045501$ [Accessed 5 Jun 2019].

40 Government of Canada SC. Physical activity, self reported, youth, 12 to 17, 2018. Available: https://www150.statcan.gc.ca/t1/tbl1/en/tv. action?pid=1310009614 [Accessed 5 Jun 2019].

41 Murdoch JR, Lloyd CM. Chronic inflammation and asthma. Mutat Res Mol Mech Mutagen 2010;690:24-39.

42 Vitcu A, Lungu E, Vitcu L, et al. Multi-stage maximum variation sampling in health promotion programs' evaluation. Journal of Preventive Medicine 2007;15:5-18.

43 Rawson KA, Gunstad J, Hughes J, et al. The meter: a brief, self-administered measure of health literacy. J Gen Intern Med 2010;25:67-71

44 Harris PA, Taylor R, Thielke R, et al. Research electronic data capture (REDCap)--a metadata-driven methodology and workflow process for providing translational research informatics support. J Biomed Inform 2009;42:377-81.

45 Greenhalgh T, Robert G, Macfarlane F, et al. Diffusion of innovations in service organizations: systematic review and recommendations. Milbank Q 2004;82:581-629.

46 Huijg JM, Crone MR, Verheijden MW, et al. Factors influencing the adoption, implementation, and continuation of physical activity interventions in primary health care: a Delphi study. BMC Fam Pract 2013;14:142.

47 Bowen S, Sustar H, Wolstenholme D, et al. Engaging teenagers productively in service design. Int $J$ Child Comput Interact 2013:1:71-81.

48 Gallagher M, Hares T, Spencer J, et al. The nominal group technique: a research tool for general practice? Fam Pract 1993;10:76-81.

49 Atkins L, Francis J, Islam R, et al. A guide to using the theoretical domains framework of behaviour change to investigate implementation problems. Implement Sci 2017;12:77.

50 Truong KN, Hayes GR, Abowd GD. Storyboarding: an empirical determination of best practices and effective guidelines. New York, NY, USA: ACM: Proceedings of the 6th Conference on Designing Interactive Systems, 2006: 12-21.

51 Cane J, Richardson M, Johnston M, et al. From Lists of behaviour change techniques (BCTs) to structured hierarchies: comparison of two methods of developing a hierarchy of BCTs. Br J Health Psychol 2015;20:130-50.

52 GWK H. Examining perceptions and attitudes: a review of Likert-Type scales versus Q-Methodology. West J Nurs Res 2017;39:674-89.

53 Moore A, Brouwer M, Straus S, et al. Advancing patient and public involvement in guidelines development. Canadian Taskforce on Preventative Health Care 2015.

54 Brown H. A guide to capturing and using patient, public and service user feedback effectively. Health Services Management Centre: University of Birmingham, 2009.

55 Hamilton CB, Hoens AM, McQuitty S, et al. Development and pre-testing of the patient engagement in research scale (PEIRS) to assess the quality of engagement from a patient perspective. PLOS One 2018;13:e0206588.

56 Hsieh H-F, Shannon SE. Three approaches to qualitative content analysis. Qual Health Res 2005;15:1277-88.

57 Kushniruk AW, Patel VL. Cognitive and usability engineering methods for the evaluation of clinical information systems. J Biomed Inform 2004;37:56-76.

58 Stinson J, Gupta A, Dupuis F, et al. Usability testing of an online selfmanagement program for adolescents with cancer. $J$ Pediatr Oncol Nurs 2015;32:70-82.

59 O'Malley G, Dowdall G, Burls A, et al. Exploring the usability of a mobile APP for adolescent obesity management. JMIR Mhealth Uhealth 2014:2:e29.

$60 \mathrm{Yu} \mathrm{CH}$, Parsons JA, Hall S, et al. User-centered design of a webbased self-management site for individuals with type 2 diabetes providing a sense of control and community. BMC Med Inform Decis Mak 2014;14:60

61 Nielsen J. Getting Usability Used. In: Nordby K, Helmersen P, Gilmore DJ, et al, eds. Human-Computer interaction. Springer US, 1995: 3-12.

62 Gibson PA, Ruby C, Craig MD. A health/patient education database for family practice. Bull Med Libr Assoc 1991;79:357-69.

63 Carter N, Bryant-Lukosius D, DiCenso A, et al. The use of triangulation in qualitative research. Oncol Nurs Forum 2014;41:545-7.

64 Hackett CL, Mulvale G, Miatello A. Co-designing for quality: creating a user-driven tool to improve quality in youth mental health services. Health Expect 2018;21:1013-23.

65 Hjelmfors L, Strömberg A, Friedrichsen M, et al. Using co-design to develop an intervention to improve communication about the heart failure trajectory and end-of-life care. BMC Palliat Care 2018:17:85.

66 Jessen S, Mirkovic J, Ruland CM. Creating Gameful design in mHealth: a participatory Co-Design approach. JMIR Mhealth Uhealth 2018;6:e11579.

67 Jessup RL, Osborne RH, Buchbinder R, et al. Using co-design to develop interventions to address health literacy needs in a hospitalised population. BMC Health Serv Res 2018;18.

68 Marent B, Henwood F, Darking M, et al. Ambivalence in digital health: Co-designing an mHealth platform for HIV care. Soc Sci Med 2018;215:133-41.

69 Masterson-Algar P, Williams S, Burton CR, et al. Getting back to life after stroke: co-designing a peer-led coaching intervention to enable stroke survivors to rebuild a meaningful life after stroke. Disabil Rehabil 2018:1-14.

70 Mayrhofer AM, Mathie E, McKeown J, et al. Young onset dementia: public involvement in co-designing community-based support. Dementia 2020:19:1051-66.

71 Persaud N, Davidson M, Charter D. Community members codesigning a trial of medication access. CMAJ 2018;190:S44-5.

72 Schoultz M, Macaden L, Watson AJM. Co-designing inflammatory bowel disease (IBD) services in Scotland: findings from a nationwide survey. BMC Health Serv Res 2016;16:231. 
73 Seid M, Dellal G, Peterson LE, et al. Co-Designing a collaborative chronic care network (C3N) for inflammatory bowel disease: development of methods. JMIR Hum Factors 2018;5:e8.

74 Owens C, Farrand P, Darvill R, et al. Involving service users in intervention design: a participatory approach to developing a textmessaging intervention to reduce repetition of self-harm. Health Expect 2011;14:285-95.

75 Campbell NC, Murray E, Darbyshire J, et al. Designing and evaluating complex interventions to improve health care. BMJ 2007;334:455-9.
76 Dent-Spargo R. Using Self-Determination theory to support CoDesign activities. CEUR Workshop Proc 2018.

77 Johansson-Sköldberg U, Woodilla J, Çetinkaya M. Design thinking: past, present and possible futures. Creativity and Innovation Management 2013;22:121-46.

78 Bowen S, McSeveny K, Lockley E, et al. How was it for you? experiences of participatory design in the UK health service. CoDesign 2013;9:230-46. 